\title{
Harnessing Connected Health to Improve Hospital Discharge and Patient Outcomes for Every Generation: Insights from Piloting the Corrie Platform in Post-MI Recovery
}

Lochan Shah $^{1}$; Francoise Marvel ${ }^{1}$, MD; Erin Spaulding ${ }^{2}$; William Yang ${ }^{1}$, MD; Helen Xun ${ }^{1}$; Farhan Merali ${ }^{1}$, MD; Matthias Lee ${ }^{3}$, MD; Seth Martin ${ }^{1}$, MD

\footnotetext{
${ }^{1}$ Johns Hopkins University, School of Medicine, Baltimore, MD, United States

${ }^{2}$ Johns Hopkins University, School of Nursing, Baltimore, MD, United States

${ }^{3}$ Johns Hopkins University, Baltimore, MD, United States
}

Corresponding Author:

Lochan Shah

Johns Hopkins University

School of Medicine

733 N Broadway

Baltimore, MD,

United States

Phone: 8587351997

Email: 1shah5@jhmi.edu

\begin{abstract}
Background: Rapid adoption of smartphones has led to an increasing interest in connected health interventions for clinical care and remote monitoring. However, there is a large disconnect between clinical patient populations and the digital health literature, as most digital health work has occurred outside the healthcare system in younger, healthy patients. The applicability of many innovative connected health approaches across generations, particularly in older patients presenting with coronary heart disease, is a topic in strong need of further study. Our interdisciplinary team of clinicians and engineers built the 1st cardiology CareKit application ("Corrie"), focused on improving hospital discharge and empowering patients from all generations through an optimal recovery from acute myocardial infarction. We now report on our initial piloting experience with a focus on use and outcomes across generations.
\end{abstract}

Objective: In this interim analysis of the first 47 patients enrolled in the Myocardial infarction COmbined device Recovery Enhancement (MiCORE) Pilot Study, we sought to assess the feasibility and uptake of a connected health platform in patients hospitalized with acute myocardial infarction (AMI) and to assess 30-day post-discharge readmission risk across generations.

Methods: This prospective observational study enrolled acute MI patients from the Cardiac Units at two sites-Johns Hopkins Bayview and Johns Hopkins Hospital. The intervention, consisting of an Apple CareKit smartphone application ("Corrie app”) and Apple smartwatch, allowed patients to develop medication self-management skills, coordinate follow-up appointments, learn about critical preventative cardiology topics via videos, and connect with health resources across the continuum of care. AMI patients were enrolled during admission and used the intervention from enrollment through 30-days post-discharge. The intervention was developed with substantial efforts to optimize user-centered design. 30-day readmission rate was assessed by chart review of electronic medical record and CRISP regional data. Descriptive statistics were performed to examine user demographics.

Results: 30 -day readmission rates in Corrie-enrolled patients $(0 \%, \mathrm{~N}=47)$ were significantly lower than the Johns Hopkins historical control $(19 \%, \mathrm{~N}=200 ; P<0.001) .70 .2 \%$ of Corrie patients were men and $29.8 \%$ were women, with roughly half of the patients above the age of $60(46.8 \%)$. The mean age was 58 (SD 11), with a full range from 32-76 years. Patients represented all generations: $8.5 \%$ were from the Silent Generation (born before 1945), 55.3\% were Baby Boomers (born 1945-1964), 31.9\% were from Generation X (born 1964-1980) and 4.3\% were Millennials/Generation Y (born 1980-1999). The proportion of White/Caucasian participants was 68.1\%, Black/African American was 14.9\%, American Indian/Alaska native was 2.1\%, Asian was $4.2 \%$, and unknown/other was $10.6 \%$. Examining socioeconomic status, $10.7 \%$ had Medicaid, $8.5 \%$ had Medicare, $78.7 \%$ had private insurance, and $2.1 \%$ had no insurance. The average median income according to patient zip codes was $\$ 74,717$.

Conclusions: In our study that stands out for its focus on deployment of patient-centered connected health in the clinical care setting, the Corrie platform shows evidence of uptake, feasibility, and effectiveness of digital health interventions across patients 
of multiple generations and demographics. Additional studies are ongoing to evaluate differential user engagement between demographic groups with respect to specific features of the smartphone-application based intervention.

(iproc 2017;3(1):e27) doi: 10.2196/iproc.8426

\section{KEYWORDS}

myocardial infarction; smartphone application; wearables; smartwatch; self-management; hospital discharge; clinical care; patient empowerment; cardiology app

\section{Multimedia Appendix 1}

Full poster.

[PDF File (Adobe PDF File), 59MB-Multimedia Appendix 1]

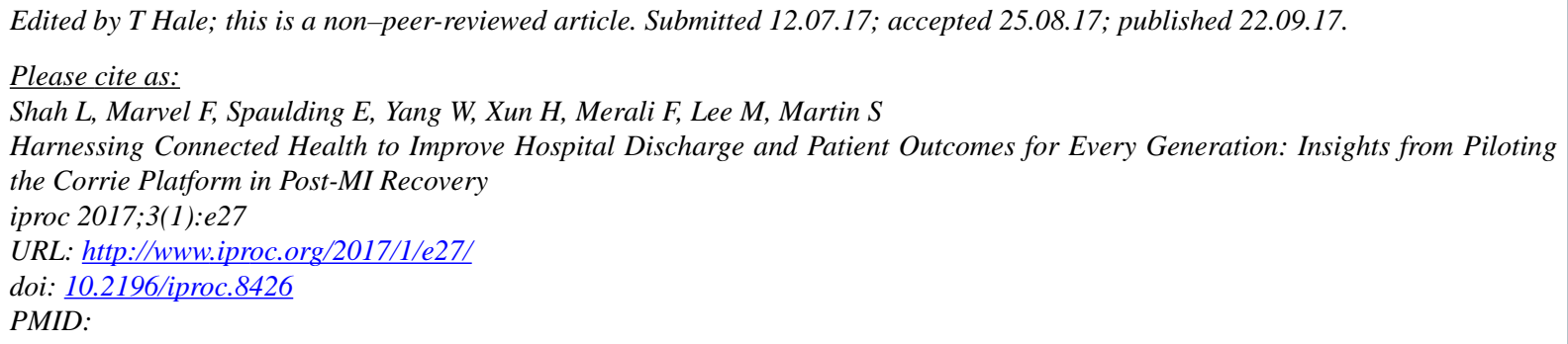

CLochan Shah, Francoise Marvel, Erin Spaulding, William Yang, Helen Xun, Farhan Merali, Matthias Lee, Seth Martin. Originally published in Iproceedings (http://www.iproc.org), 22.09.2017. This is an open-access article distributed under the terms of the Creative Commons Attribution License (https://creativecommons.org/licenses/by/4.0/), which permits unrestricted use, distribution, and reproduction in any medium, provided the original work, first published in Iproceedings, is properly cited. The complete bibliographic information, a link to the original publication on http://www.iproc.org/, as well as this copyright and license information must be included. 